Biomedical Engineering / Biomedizinische Technik I Ahead of Print

\title{
Classification of standing and sitting phases based on in-socket piezoelectric sensors in a transfemoral amputee
}

\author{
Tawfik Yahya, Nur Azah Hamzaid $\backsim$, Sadeeq Ali, Farahiyah Jasni and Hanie Nadia Shasmin
}

DOI: https://doi.org/10.1515/bmt-2018-0249 I Published online: 27 May 2020

\section{Abstract}

A transfemoral prosthesis is required to assist amputees to perform the activity of daily living (ADL). The passive prosthesis has some drawbacks such as utilization of high metabolic energy. In contrast, the active prosthesis consumes less metabolic energy and offers better performance. However, the recent active prosthesis uses surface electromyography as its sensory system which has weak signals with microvolt-level intensity and requires a lot of computation to extract features. This paper focuses on recognizing different phases of sitting and standing of a transfemoral amputee using in-socket piezoelectric-based sensors. 15 piezoelectric film sensors were embedded in the inner socket wall adjacent to the most active regions of the agonist and antagonist knee extensor and flexor muscles, i. e. region with the highest level of muscle contractions of the quadriceps and hamstring. A male transfemoral amputee wore the instrumented socket and was instructed to perform several sitting and standing phases using an armless chair. Data was collected from the 15 embedded sensors and went through signal conditioning circuits. The overlapping analysis window technique was used to segment the data using different window lengths. Fifteen timedomain and frequency-domain features were extracted and new feature sets were obtained based on the feature performance. Eight of the common pattern recognition multiclass classifiers were evaluated and compared. Regression analysis was used to investigate the impact of the number of features and the window lengths on the classifiers' accuracies, and Analysis of Variance (ANOVA) was used to test significant differences in the classifiers' 
performances. The classification accuracy was calculated using k-fold cross-validation method, and $20 \%$ of the data set was held out for testing the optimal classifier. The results showed that the feature set (FS-5) consisting of the root mean square (RMS) and the number of peaks (NP) achieved the highest classification accuracy in five classifiers. Support vector machine (SVM) with cubic kernel proved to be the optimal classifier, and it achieved a classification accuracy of $98.33 \%$ using the test data set. Obtaining high classification accuracy using only two time-domain features would significantly reduce the processing time of controlling a prosthesis and eliminate substantial delay. The proposed in-socket sensors used to detect sit-to-stand and stand-to-sit movements could be further integrated with an active knee joint actuation system to produce powered assistance during energy-demanding activities such as sit-to-stand and stair climbing. In future, the system could also be used to accurately predict the intended movement based on their residual limb's muscle and mechanical behaviour as detected by the in-socket sensory system.

Keywords: feature selection; in-socket sensory system; prosthetic leg; window length

\section{PURCHASE ARTICLE}

Get instant unlimited access to the article.

\section{$€ 30.00$}

\section{ADD TO CART}

or

\section{REDEEM TOKEN >}

or

\section{LOG IN}

Already have access? Please log in.

Email 
or

\section{ARTICLE INFORMATION}

Corresponding author: Nur Azah Hamzaid, Biomedical Engineering Department, Faculty of Engineering,

University of Malaya, 50603, Kuala Lumpur, Malaysia; and Centre for Applied Biomechanics, University of Malaya, 50603, Kuala Lumpur, Malaysia, E-mail:

Received: 2018-12-20

Accepted: 2020-02-14

Published Online: 2020-05-27

Citation Information: Biomedical Engineering / Biomedizinische Technik , 000010151520180249, eISSN 1862278X, ISSN 0013-5585, DOI: https://doi.org/10.1515/bmt-2018-0249.

(C) 2020 Walter de Gruyter GmbH, Berlin/Boston.

Get Permission

\section{JOURNAL + ISSUES}

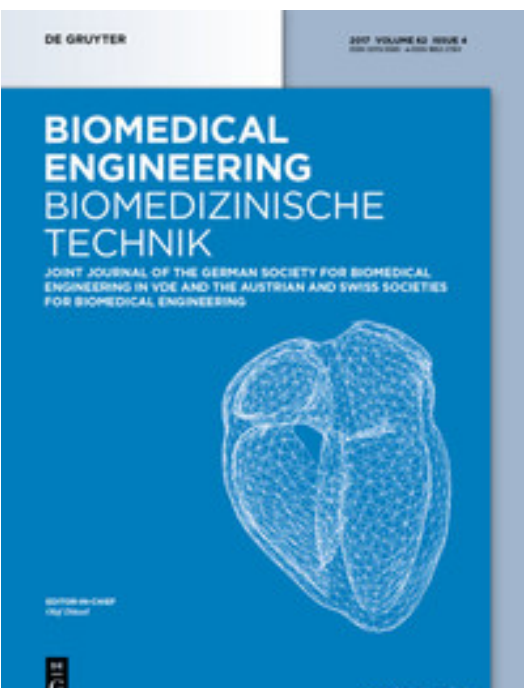

Biomedical Engineering / Biomedizinische Technik 


\section{三: Ahead of Print}

\section{DETAILS}

Biomedical Engineering / Biomedizinische Technik (BMT) is a high-quality forum for the exchange of knowledge in the fields of biomedical engineering, medical information technology and biotechnology/bioengineering. BMT addresses engineers, natural scientists, and clinicians working in research, industry, or clinical practice. All articles are peer-reviewed.

Online ISSN: 1862-278X

First published: 01 Jan 1956

Language: English

Publisher: De Gruyter

\section{SEARCH}

Search within Journal...

$\bigcirc$ Issue $\bigcirc$ Journal

Volume Issue Page Q

\section{AUTHENTICATED BY}

International Islamic University Malaysia (IIUM) 


\section{IMPRINTS}

Birkhäuser

De Gruyter Mouton

De Gruyter Oldenbourg

De Gruyter Saur

Deutscher Kunstverlag

Edition Klaus Schwarz

Jovis

\section{CONTACT \& SERVICE}

Help Center

Contact

Career

Our Locations

Imprint

\section{STAY IN TOUCH}

\section{$f y 0$}

$\mathrm{B} \log s$

Newsletter

Privacy Policy Terms and Conditions Disclaimer

(C) Walter de Gruyter GmbH 2020

Authenticated by: International Islamic University Malaysia (IIUM) 